\title{
TERAPIA A TRAVÉS DE LA DANZA Y EL MOVIMIENTO EN MUJERES CON CÁNCER DE MAMA: EL IMPACTO PSICOFÍSICO DEL PROCESO EMPÁTICO
}

\author{
DANCE MOVEMENT THERAPY AND WOMEN WITH BREAST CANCER: THE \\ PSYCHOPHYSICAL IMPACT OF THE EMPATHIC PROCESS
}

\author{
Alicia Blázquez', Judit Nierga² y Casimiro Javierre? \\ 1 Departamento de Ciencias Fisiológicas II. Facultad de Medicina. Universidad de Barcelona. IDIBELL. L'Hospitalet. \\ Barcelona. \\ 2 Hospital de Día de Adultos. Parque Sanitario Martí i Julià. IES. Salt. Girona.
}

Resumen

Objetivos: describir el proceso terapéutico y la posible influencia de las neuronas espejo en la empatía kinestésica empleando la terapia a través de la danza y el movimiento en el cáncer de mama.

Método: se utilizó un estudio de caso. Una paciente diagnosticada con cáncer de mama desde hace dos años fue derivada al Departamento de Ciencias fisiológicas II de la Universidad de Barcelona. Ella asistió a una intervención con terapia a través de la danza y el movimiento durante 5 meses ( 1 hora semanal, 20 sesiones). Su historia clínica y las anotaciones tanto de ella como del terapeuta fueron revisadas.

Resultado: La paciente mostró una mejora en su bienestar psicofísico percibido después de participar en el programa de terapia a través de la danza y el movimiento. Este tipo de intervención facilitó la recogida de información tanto a nivel físico como psicológico de la paciente. El proceso empático fue relevante para ello.

Conclusiones: El bienestar percibido y expresado por la paciente a lo largo de las sesiones permite entrever la importancia clínica de la terapia a través de la danza y el movimiento. Una futura propuesta sería realizar dicha intervención en un contexto grupal,
Abstract

Objectives: To describe the therapeutic process and possible influence of mirror neurons in kinesthetic empathy using dance movement therapy for breast cancer.

Method: Case study methodology was used. A patient diagnosed with breast cancer two years previously was referred to the Department of Physiological Sciences II, University of Barcelona. She attended a dance movement therapy intervention for 5 months ( 1 hour per week, 20 sessions). The patient's medical history and a therapist's records were reviewed.

Result: The patient showed an improvement in perceived mental and physical well-being after participating in the program of dance movement therapy. This type of intervention facilitated the collection of physical and psychological patient information. Empathy was vital to this process.

Conclusions: The perceived well-being expressed by the patient throughout the sessions enhances the clinical significance of dance movement therapy. It is recommended that a trial is undertaken to assess the effectiveness of dance movement therapy on breast cancer patients' mental and physical well-being.

Key words: Breast cancer, dance movement therapy, Kinestesic empathy, mirror neurons.

\section{Correspondencia a:}

Alicia Blázquez

Departamento de Ciencias Fisiológicas II. Facultad de Medicina. Universidad de Barcelona.

Ctra. Feixa Llarga s/n. 08.907. L'Hospitalet de Llobregat. Barcelona.

E-mail: ablazquez@ub.edu 
ampliando y diversificando el proceso empático al añadir al binomio terapeuta-paciente el de paciente-paciente.

Palabras clave: Cáncer de mama, danza movimiento terapia, empatía kinestésica, neuronas espejo.

\section{INTRODUCCIÓN}

Cada vez son más los estudios que asocian la terapia a través del movimiento y la danza (DMT) con mejoras en pacientes diagnosticados con cáncer ${ }^{(1-3)}$. ¿Cómo podría definirse la DMT? Como el "Uso psicoterapéutico del movimiento y la danza dentro de un proceso que persigue la integración psicofísica del individuo ${ }^{\prime \prime 4,5)}$. La DMT emplea la integración dinámica del cuerpo y la mente a través del uso de experiencias de movimiento, la expresión creativa y la integración de técnicas de relajación e imaginería para facilitar la comunicación y el trabajo expresivo.

Los cinco principios teóricos en los que se basa la DMT serían: 1) Cuerpo-mente están en constante interacción recíproca ${ }^{(6,7)}$. En la DMT se anima al paciente a conectar la comprensión verbal con la experiencia de movimiento, posibilitando la libre asociación e interpretación. 2) El movimiento refleja la personalidad. La DMT no es moverse más libre o perfectamente, sino usar la experimentación para explorar nuevas maneras de ser y de sentir, y para ganar acceso a sentimientos que no pueden ser verbalizados $^{(8)}$. 3) La relación del danzaterapeuta y el paciente es central para la efectividad del enfoque. "Espejeando", sincronizando, amplificando, interactuando con el movimiento del paciente. 4) Tanto el movimiento, como los sueños, los dibujos, los garabatos, deslices de la lengua y la libre asociación, pueden ser evidencias de procesos no conscientes ${ }^{(9)}$. Al referirse, en su libro "The thinking body" al lenguaje corporal, Mabel Elsworth Todd escribe: "a menudo el cuerpo dice con claridad lo que la lengua se niega a enunciar"(10). 5) El acto de crear un movimiento a través de la improvisación es inherentemente terapéutico desde que permite al individuo experimentar maneras originales/novedosas de movimiento, que generan una nueva experiencia de estar en el mundo.

En cáncer, la DMT y otros métodos creativos potencian en los individuos el expresar necesidades y sentimientos tanto a nivel verbal como no verbal, ayudando a las personas a reconectar con su cuerpo, especialmente tras la extirpación del tumor ${ }^{(2,11-13)}$. El creciente número de estudios de DMT en pacientes con cáncer, especialmente en mujeres con cáncer de mama, enfatiza su importancia al ser una aproximación integradora que puede mejorar la calidad de vida de la paciente $^{(1,14,15)}$. La DMT fortalece el sistema inmune a través de la acción muscular y de la activación de diversas respuestas fisiológicas y, también, puede eliminar la tensión y otras condiciones de discapacidad como resultado de la situación de estrés ${ }^{(1,16)}$. De hecho, Dibbel-hope comprobó mejorías en el bienestar psicológico de mujeres con cáncer de mama, disminuyendo aspectos relacionados con la tensión, la ansiedad, la depresión, la ira y la confusión-perplejidad ante el diagnóstico ${ }^{(11)}$.

En DMT, el terapeuta tiene una participación activa, siendo sus respuestas, incluidas las somáticas parte del encuentro terapeuta-paciente $^{(17)}$. En la DMT el cuerpo y el movimiento son el lenguaje primario. Por ello la comunicación no verbal es tan importante y aspectos corporales de la 
relación terapéutica deben ser tenidos en cuenta. Varios teóricos sobre las implicaciones corporales en la terapia insisten en que la contratransferencia somática (CTS) (todos aquellos sentimientos que el analista experimenta en su cuerpo a través de la relación terapéutica con el paciente) es significativa en la relación psicoterapéutica. No sólo es una herramienta para entender y manejar las dinámicas durante la sesión sino que además permite enfatizar el proceso terapéutico ${ }^{(18-25)}$. El cuerpo del terapeuta sintoniza con el cuerpo de la paciente permitiendo al inconsciente manifestarse a través de la comunicación verbal y no verbal ${ }^{(26)}$. De ahí la importancia de la "empatía kinestésica". El concepto de "empatía kinestésica" fue mencionado por primera vez por Miriam Roskin Berger en 1956 en una tesis que no fue publicada hasta $1989^{(27)}$. Dosamantes-Alperson en 1984 la describía como la reproducción de los movimientos corporales del paciente en el cuerpo del terapeuta, lo que le permitiría a este último sentir y responder a los estados emocionales del paciente ${ }^{(28)}$. Eso daría lugar a un proceso de descubrimiento y co-construcción centrado en el desarrollo del sentido del self del paciente ${ }^{(18,19)}$.

En relación con la empatía, Jean Decety propuso una anatomía basada en ella. La resonancia motriz sería producida por las "neuronas espejo" del córtex motor y del córtex parietal que se activan tanto cuando el sujeto actúa, como cuando observa a otro sujeto realizar la acción. Las neuronas espejo fueron un descubrimiento casual del equipo dirigido por Giacomo Rizzolati de la Universidad de Parma ${ }^{(29,30)}$. Desde entonces se ha mejorado el entendimiento sobre el sistema neural y se ha permitido traducir los conceptos básicos a aplicaciones clínicas iniciales tanto en psiquiatría como en neurología ${ }^{(31)}$. El sistema de neuronas espejo en humanos está directamente relacionado con la imitación de movimientos simples ${ }^{(32)}$, el aprendizaje imitativo de habilidades complejas $^{(33)} y$ en la percepción de acciones comunicativas $^{(34)}$. Las propiedades de estas neuronas proveen un código flexible de acción de uno mismo y de los otros. Dicha flexibilidad es importante en las interacciones sociales $^{(35)}$ y en este caso probablemente tenga un peso importante en la relación terapeuta-paciente. En referencia a ello, la empatía permitiría al terapeuta "introducir$\mathrm{se}^{\prime \prime}$ en la experiencia del paciente, permaneciendo, sin embargo, emocionalmente independiente $^{(36)}$.

El objetivo principal de este artículo es la presentación de un caso clínico donde se muestra la mejora percibida por una paciente diagnosticada con cáncer de mama tras una intervención en DMT. A través de una exposición cronológica de las aportaciones escritas tanto de ella como de su terapeuta, se enfatiza la importancia de la relación terapeuta-paciente dentro de este nuevo abordaje terapéutico.

\section{MÉTODO}

Una paciente diagnosticada con cáncer de mama desde hace dos años, habiendo recibido quimioterapia y radioterapia consecutivamente, fue derivada al Departamento de Ciencias fisiológicas II de la Universidad de Barcelona.

Carmen es una mujer de 32 años, peluquera aunque en baja laboral. Está separada desde hace años y actualmente se encuentra en otra relación de pareja. Tiene una hija de su anterior matrimonio. La relación con su familia de origen es compleja: su madre está en una residencia por demencia senil y con su padre y hermano la relación es distante.

La demanda inicial de la paciente al incorporarse al programa de DMT fue por "curiosidad" y como una posibilidad de autoconocimiento. Además verbalizó que le gustaba la danza. 
Las sesiones fueron llevadas a cabo de febrero 2010 a julio 2010, con una sesión de una hora cada semana, con un total de 20 sesiones. Se dispuso de un espacio habilitado con luz natural y el material necesario para el desarrollo del programa (colchonetas, pelotas, sillas, cuerdas, cintas, telas...).

A la hora de valorar la información proporcionada por la paciente durante la intervención con DMT, se tuvo en cuenta que la DMT tiene 4 características que la distinguen de otras intervenciones de apoyo psicosociales en un contexto asistencial biomédico $^{(37)}$ :

1- La incorporación del cuerpo en el proceso psicoterapéutico.

2- La construcción de una relación con el cuerpo de la paciente.

3- La promoción del establecimiento o reestablecimiento de una relación positiva entre la paciente y su cuerpo.
4- El uso de la expresión del movimiento creativo para promover la expresión de salud.

Basándonos en ello, los objetivos principales durantes las 20 sesiones fueron los siguientes (ver figura 1 ).

Si bien todos los objetivos se tuvieron en cuenta a lo largo de las sesiones, algunos eran más relevantes según el momento de la sesión. Cada sesión estaba estructurada tal como se expone en la tabla 1.

En definitiva, la DMT constituye una modalidad de tratamiento para intervenir en el individuo de forma integradora, combinando aspectos como el trabajo con respiración, ejercicios de relajación y de conciencia corporal, la creatividad a través de la interacción social... ${ }^{(38)}$.

La recogida de datos se hizo cualitativamente a través de:

- La historia clínica de la paciente.

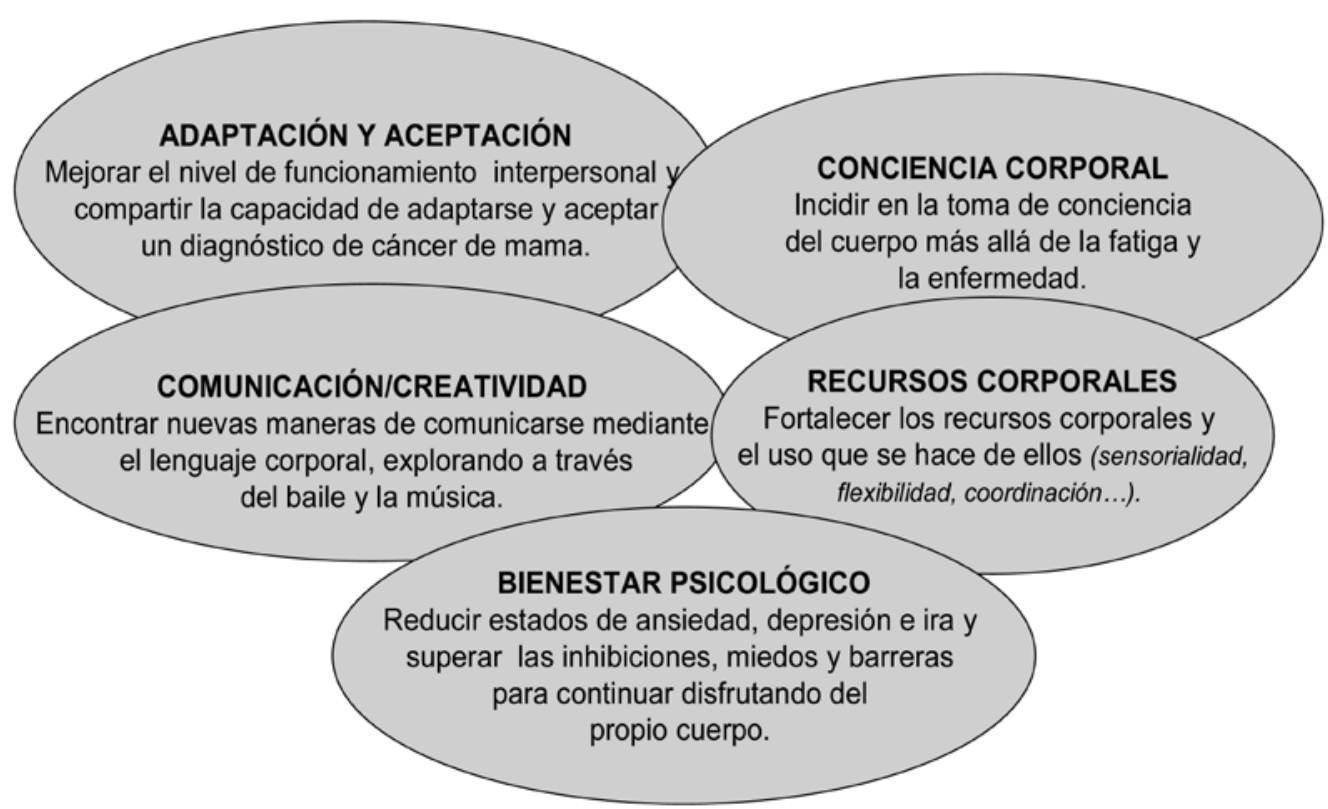


- Un registro escrito a lo largo del programa de la paciente participante, después de cada sesión, sobre sus impresiones, sentimientos, pensamientos.

- Notas personales a lo largo del programa de la danzaterapeuta (también psicoterapeuta) sobre la evolución de la paciente.

En un estudio de Petrone, además de seguir un modelo similar al nuestro ya que organizaba las sesiones en base a un calentamiento, desarrollo y cierre $^{(39)}$; opinaba que el diario de la experiencia de los participantes permitía describir los beneficios del programa, especialmente para la reducción del aislamiento, daba apoyo para la expresión de las emociones y permitía mejorar las actitudes hacía la enfermedad $^{(12)}$.

\section{RESULTADOS}

A continuación se presentan cronológicamente extractos de los comentarios tan- to de la paciente (en cursiva) como de la danzaterapeuta.

\section{5/02/2010}

Probamos con el llevar y ser llevada (una con los ojos cerrados, la otra guiando y viceversa). Se cansa mucho más al Ilevarme que al ser llevada. "Es como si me sintiera obligada a hacer de más", me comenta que su sentido autoexigente y su culpa influyen mucho. Debe cargar con todo. Si una persona no puede distinguir entre varios sentimientos no puede describirlos ni diferenciarlos y por tanto expresarlos en palabras $^{(40)}$. La experiencia corporal permitió a Carmen tomar más conciencia de esa distinción.

\section{$11 / 03 / 2010$}

Cuando suena la música debe caminar por el espacio (ella baila con fuerza, mar-

\section{Tabla 1. Estructura de las sesiones de DMT}

\begin{tabular}{|l|l|}
\hline ACOMODACIÓN & $\begin{array}{l}\text { Al empezar la sesión la paciente tenía un } \\
\text { espacio para expresar su estado físico y } \\
\text { emocional, sus expectativas, sus necesidades } \\
\text { (Adaptación y aceptación. Se potencia el } \\
\text { vínculo terapéutico). }\end{array}$ \\
\hline CALENTAMIENTO & $\begin{array}{l}\text { Uso de distintas técnicas sensoriales,...para } \\
\text { iniciar la toma de conciencia con el cuerpo } \\
\text { (Conciencia corporal, recursos corporales). }\end{array}$ \\
\hline DESARROLLO & $\begin{array}{l}\text { Desarrollo de la sesión a través de una } \\
\text { temática concreta: potenciar recursos } \\
\text { personales psicofísicos (Comunicación/ } \\
\text { creatividad). }\end{array}$ \\
\hline RELAJACIÓN & $\begin{array}{l}\text { Incidir en ejercicios de respiración y } \\
\text { estiramientos. Vuelta a la calma (Conciencia } \\
\text { corporal, recursos corporales). }\end{array}$ \\
\hline DEVOLUCIÓN & $\begin{array}{l}\text { Integración psicofísica valorando el significado } \\
\text { dado a los movimientos y pensamientos } \\
\text { asociados (Bienestar psicológico). }\end{array}$ \\
\hline
\end{tabular}


cando mucho el ritmo con los pies y con las palmas hacía abajo, haciendo sonido especialmente con el talón). Cuando para la música, le digo una palabra relacionada con la teoría de Rudolf Laban ${ }^{(41)}$ y de ahí a ver qué movimiento le sugiere

"Las palabras me han evocado situaciones, sentimientos vividos. He encontrado que era una buena elección de palabras, porqué todas se podían interpretar en positivo o negativo: "pesado/ligero", "contenido/libre", "lento/rápido", "directo/ indirecto": en algunas me sentía incómoda, como en el caso de "indirecto" que lo relacionaba con hacer cosas malas, a escondidas. Con "rápido" me parecía mi día a día, siempre corriendo. Con "lento" me he sentido a gusto, como entre algodones, me daban ganas de moverme poco a росо".

\section{$18 / 03 / 2010$}

Retomamos lo de la semana pasada, el cómo pasar de pesado/contenido (que ella percibía negativamente) a ligero/libre (que ella veía como positivo). Quiere empezar por lo libre "porque hay más de lo pesado" (en su vida). Mientras que en lo libre destaca la ligereza, en lo pesado hace asociaciones con un pozo en el que lleva cayéndose y saliendo desde que tiene 14 años. Está cansada de contenerse y luchar para no recaer de nuevo en una depresión. Las colchonetas están en el centro y ella empieza a empujar hacía ellas, como si no quisiera ponerse encima. $Y$ cuanto más empuja, más hacía abajo va, más se agacha, y más peso pone en sus piernas... ("sentía que una fuerza me atraía hacía el pozo y yo no quería ir hacía alli") es tanta la fuerza con la que empuja que se pone a temblar y finalmente Ilora,...y de repente se cae encima de la colchoneta. Cuando se calma, se levanta y hace el movimiento como si trepara para salir de ese pozo y con mucha fuerza, con mucho dolor, con cara de agotamiento absoluto logra hacerlo y se aparta de ese pozo.

"Cuando bailaba, podía ver el pozo y su alrededor con colores y todo: el pozo de tierra gris, casi negra; el cielo oscuro, alrededor fuera del pozo aridez, sin plantas, seco, la tierra agrietada, marrón oscuro y... estaba sola, muy sola, tenía que hacer un gran esfuerzo para mantenerme fuera, sentía que debía hacer mucha fuerza, que no podía más, que quería alejarme del pozo y no...Me sentía representando mi vida interna. ¿Cosas que he aprendido?: iestoy fuera del pozo! Llevo días relativizando la ansiedad y todo va mejor. Todos tenemos problemas, todos tenemos fantasmas, todos tenemos monstruos en nuestro interior, pero si están fuera dejan de ser fantasmas, no son tan monstruos y se pueden mejorar, y yo lo he hecho y lo hago".

La DMT propone un dispositivo teóricoclínico que permite operar allí donde las palabras no son suficiente vía de contacto y encuentro, favoreciendo la instauración de un tipo de transferencia que opera en un nivel psicocorporal. Se trata de rescatar las vivencias que quedaron congeladas en el cuerpo a través de, por ejemplo, contracturas crónicas que inhibieron movimientos. A través de la DMT Carmen pudo acceder a más símbolos, obtuvo una dimensión interna más profunda. Ahora era capaz de relacionar emociones evocadas en el proceso empático con el terapeuta. Sus experiencias internas y externas seguían en el proceso de diferenciarse.

\section{1/04/2010}

Inicio una secuencia con ella que me está resultando muy útil con las pacientes con cáncer de mama, no sólo para trabajar el estirar la zona del pecho y las axilas (después de la operación todas sienten cierta tirantez e incomodidad al alzar los brazos) sino también para aumentar la conciencia corporal en relación a los 
conceptos de "apertura" y "cierre" (tanto literal como metafórico).

"Hemos hablado de mi ex, evidentemente aún hay rabia y resentimiento. Me gusta la apertura del pecho. He conseguido abrir más la parte alta del pecho y darme cuenta que he abierto y cerrado al subir y bajar los brazos. Ha sido muy agradable".

\section{8/04/2010}

"...concepto de "fragilidad" con "flexibilidad" y "fortaleza" con "dureza" y "rigidez", no lo había pensado nunca... quizás la sensación de fragilidad, mi miedo a tener miedo, inseguridades, dudas, pereza... está relacionada a las ganas de hacerlo todo bien, de no equivocarme, de no tener problemas, es decir, aunque muchas veces no me dé cuenta, de ser perfecta. $Y$ eso por suerte, jes imposible! Esta necesidad ha salido del hecho que me pasé mi infancia pensando que si me equivocaba o si la liaba mis padres me dejarían de querer y me abandonarían".

La diferencia de la DMT con una simple técnica de expresión es que el psicoterapeuta permite utilizar lo que ocurre en las sesiones para comprender la vida afectiva del adulto (y su "niño" interno) y así elucidar su malestar interior.

\section{$15 / 04 / 2010$}

...Le propongo que con las telas que le he dejado en el centro (distintas texturas, midas, colores) dentro de un aro que intente representar qué significaría esa "fragilidad" para ella. No la dirijo mucho porque el trabajo con Carmen siempre es fácil, tiene mucha capacidad de introspección, de reflexión. Pero esta vez se queda como inhibida, incómoda,...tal como verbaliza más tarde. Me comenta que esto tiene mucho que ver con el querer hacer las cosas bien, algo que le viene de muy pequeña.
"Lo que más me ha sorprendido ha sido el ejercicio con las telas. Representar con ellas mi fragilidad y más ahora, sin una connotación tan negativa me ha dejado estupefacta. La tela blanca, que es la que quizás más me gusta la quiero proteger, no quiero que le hagan daño desde afuera, por eso cojo las telas de lana, porque son cálidas y dulces y suaves; cojo dos piezas porque me quiero tapar por delante $y$ detrás y una solo no me alcanza. En este ejercicio me doy cuenta que hay una cosa que para mí es significativamente importante: la protección que me quiero poner es cálida, dulce, suave, tierna, ahora pienso que quiero compañía, cariño; no es rígida, dura, fría, defensiva, como un castillo como siempre me había sentido y para mi es importante...para mi fue muy bonito, porque es la primera vez que me puedo imaginar como una niña pequeña y sentirme contenta".

El desarrollo de la conciencia del self provee de la capacidad de objetivar el propio self y utilizar símbolos ${ }^{(42)}$. La conciencia del propio cuerpo y del self requiere de la capacidad de mantenerse a cierta distancia de lo que está siendo observado y de observar el propio cuerpo y el self en un contexto particular. Desde ese distanciamiento, Carmen pudo evaluar, razonar, relativizar, elegir entre varias posibilidades de acción. La conciencia del propio self-corporal le permitió, con la ayuda del terapeuta, comprender mejor su dimensión afectiva Ilevándola a un cambio psicológico.

\section{$22 / 04 / 2010$}

"También he encontrado muy interesante la interpretación que salió el otro día de niña feliz y que hoy ha salido la niña pequeña con miedo a que la riñan, y esta es la que normalmente aparece. Tengo que reconocer que cuando lo paso mal, en situaciones de presión, cuando hago errores 
reacciono como una niña pequeña asustada, me encojo, tengo miedo de las consecuencias. Evidentemente, me recuerda mi infancia, creo que la pasé con miedo a los gritos y castigos de mis padres".

La terapeuta y la paciente disponen sus cuerpos en el espacio transicional, un lugar donde la empatía kinestésica entra en juego, y quizás también las neuronas espejo. Desde ahí, van percibiendo sensaciones y emociones que asocian a imágenes, recuerdos y personas más o menos accesibles a la conciencia.

\section{9/04/2010}

Trabajamos con la idea de "empujar" con cualquier parte del cuerpo y de sentir la sensación de "ligero" mencionada anteriormente, con la idea de hacer como si "flotara".

"Otro aspecto que encuentro muy interesante a incorporar es la idea de "empujar" no como apartar algo. Me hace darme cuenta del gran esfuerzo que me supone protegerme, entiendo protegerme como apartar, como aislarme de los demás para que no me hagan daño, eso me hace sentir mucho más insegura...esta idea en contraste de "flotar", seguridad, aprovecha lo bueno que tienes, no hace falta defenderse, sólo acompañar".

\section{$13 / 05 / 2010$}

Jugamos de nuevo con el llevar y ser llevada. Esta vez su postura tiene mucho más peso, me contiene con su cuerpo y está más cerca. Ya no soy sólo yo quien apoya. Lo relaciono con tener más confianza entre nosotras y también con que su actitud corporal es mucho más firme y segura (ya no necesita que la reafirmen). Carmen suele hacer movimientos muy aéreos muy ligeros, suaves, con poco peso... en cambio al llevarme noté mucho más peso, más firmeza. Al ser llevada lo hace con facilidad, con flexibilidad. Ella misma reconoce que le cuesta ser llevada y que le ha sido más fácil esta vez.

Fue importante observar el significado simbólico de los gestos o la postura de Carmen ya que se expresaba inconscientemente a través de ellos ${ }^{(40)}$.

"Me gustó comprobar que podía dejarme llevar con facilidad, y sobretodo que me comentaste que me encontrabas más presente, más entera. Eso es lo que me impactó positivamente, lo he ido saboreando toda la semana...comienzo a recoger evidencias de "quererme"".

El terapeuta fundamenta su intervención sobre un doble soporte simbólico: la palabra, que no deja de organizar sus pensamientos, y el cuerpo que sustenta la comunicación no verbal. Es importante recibir la mirada del paciente, percibir su tono muscular y experimentar en el cuerpo las proyecciones y reacciones del paciente mientras se le acompaña y guía en su producción de sentido. Esa búsqueda de aprobación de Carmen dejó de ser desde el yopadre-terapeuta hacía su yo-hija-paciente, y pasó a una necesidad corporal-mental de reconfirmación propia. El sentir más "sano" de su self se redifinió con esas muestras de cuidado hacía si misma (ir a la peluquería "hace un año que no necesitaba irme a cortar el pelo por lo de la quimio").

\section{$20 / 05 / 2010$}

"Representar con todo el cuerpo la sensación de firmeza, de estar entera, con la música de tambores, sensación y contacto de/con la tierra. ¡Ha sido increíble! La idea de medir y repartir las fuerzas, de pasármelo bien adaptándome a las posibilidades de mi cuerpo, respetándolo, queriéndolo".

\section{3/06/2010}

Le propongo hacer con el barro su "idea" de "persona", con total libertad. 
Le digo que es una mujer muy creativa (cosa que creo firmemente ya que siempre me sorprende con lo que hace).

"Dejé que afluyeran a mi cabeza palabras, ideas que me sugerían la palabra "persona": cambio, fluir, energía,...firmeza, flotar, crecer, aprender, compartir... con el barro quería representar ideas, no necesitaba cara o cuerpo, y me encontré representando una historia... A medida que iba construyendo mi historia, el barro se iba volviendo algo vivo, lo iba queriendo. En terminar lo hice como cuando hago un masaje con pasadas suaves de los dedos por toda su superficie. Primero quería incorporarlo a mí, era como si fuera tanto yo, que quería que formara parte de mí. Con el movimiento quise representar serenidad, calma. La sensación que tengo últimamente. Tengo ganas de disfrutar del trabajo hecho conmigo misma, dejar asentar y consolidar los cambios. Recuerdo y quiero recordar la frase que después comentamos. NO HACE FALTA HACER GRANDES MOVIMIENTOS, SÓLO CAMINAR TRANQUILAMENTE. Durante este ejercicio también quería representar firmeza tierna con las manos, tengo ganas de poderlo incorporar, no hace falta tocar con inseguridad para transmitir fluidez $y$ ternura...para mí representa todo lo que puede ofrecer el entorno, las experiencias que puedes vivir aún, por lo que me moví de diferentes maneras y con todo el cuerpo. Representé algunas de alegres, divertidas, diferentes; otras dolorosas, tristes,... pero siempre había más".

\section{1/07/2010}

Lo que sí me ha impresionado ha sido lo que he sentido, lo que he hecho (ir pasando por 4 músicas distintas que "representaran" el aire, agua, fuego y tierra. Le pregunto con cual le gustaría empezar pero no se decide. Simplemente debe tratar de moverse como ella sienta ese elemento, sea como el mismo o bien dentro de él. Que perciba con cual se siente más o menos cómoda, qué es lo que observa...como responde su cuerpo a todo eso) la tierra ha comenzado a tener importancia para mí a partir de hacer yoga y fue un descubrimiento agradable pero, siempre lo había centrado a los pies y a las piernas. Esta vez, a partir de lo que hemos ido trabajando, he querido experimentar la tierra con todo el cuerpo, ha sido fantástico.

Vimos la filmación (la grabo para observar juntas su movimiento) de mi danza sobre los 4 elementos: el aire, la tierra, el agua y el fuego. Fue increíble. Comencé con mucha vergüenza al verme bailar. Desde pequeña he tenido la imagen de gorda, torpe, fea, pero fue una auténtica y preciosa sorpresa. Me vi creando, haciendo algo muy bonito, algo bello, que era hermoso de ver, me veía ágil, guapa, sensible, transmitiendo el sentimiento, auténtica, como nunca me hubiera imaginado y además fluyendo, acompañando suavemente, fue realmente emocionante para mi".

\section{DISCUSIÓN Y CONCLUSIONES}

A través del análisis de este caso hemos observado que la DMT ha permitido recrear las emociones y recuerdos que han sido reprimidos y transformarlos en experiencias concientes que la paciente pudo expresar gradualmente. De esa manera, al hacerse más visibles, la terapeuta tuvo la oportunidad de rescatarlas conectando con la paciente. Carmen, una paciente con cáncer de mama, pudo reencontrarse y rehabilitarse. En la situación terapéutica, la danza y el movimiento fueron principalmente un lugar de encuentro entre la paciente y la terapeuta. La experiencia mutua, el compartir de movimientos (espejeando) permitió a la paciente ampliar su repertorio. Carmen pudo experimentar 
placer y alegría en la DMT, después de dejarse "atravesar" por el dolor, y gracias a ello reconectar con su propio cuerpo(43).

La empatía sería ponerse en el lugar del otro sin experimentar necesariamente sus emociones, como cuando anticipamos las reacciones de alguien. La empatía no sería ni identificación, ni contagio, ni fusión. Sería el equivalente al concepto que actualmente se utiliza como "intersubjetividad" y se relacionaría con el conocimiento del otro, el compartir su estado emocional, tomar su perspectiva subjetiva y tener ante él una respuesta afectiva. Se diferenciaría de "simpatía", que es experimentar las emociones del otro sin necesariamente ponerse en su lugar. Se puede ser empático sin sentir simpatía y viceversa. Por ello, consideramos que el papel del terapeuta era el de experimentar las sensaciones y las emociones de su cliente y comunicarle esta comprensión ${ }^{(32)}$. Para Rogers, el proceso de empatía se define como "percibir el cuadro de referencia interno del otro tan precisamente como sea posible, con los componentes emocionales y las significaciones que le pertenecen como si se fuera esa persona, pero sin perder nunca de vista esa condición de "como $\mathrm{si}^{\prime \prime}$. Relacionándolo de nuevo con aspectos neurales, el descubrimiento de la neuronas espejo en monos ${ }^{(29,44)}$ y de los mecanismos de espejeo en el cerebro humano ${ }^{(31,45)}$ demuestra que los mismos substratos neurales son activados cuando algún acto expresivo es tanto ejecutada como percebida ${ }^{(46)}$. En un estudio de RMNf Carr y su grupo mostraron que tanto la observación como la imitación de la expresión facial de las emociones activa los mismas estructuras cerebrales, incluyendo el córtex premotor ventral, la insula y la amigdala ${ }^{(47)}$.

Teniendo en cuenta todo ello, el desarrollo de la intersubjetividad sería de importancia en la DMT, ya que permite un marco compartido de significados $y$ objetivos de la comunicación como son los gestos, las posturas o las expresiones faciales ${ }^{(48,49)}$. Relacionándolo con el espejeo que se produce entre el paciente y el danzaterapeuta durante la sesión, eso implica por parte del terapeuta la capacidad de sentir un estado afectivo compartido sin necesariamente imitar exactamente la expresión comportamental del otro ${ }^{(48)}$. De ahí que el mismo tipo de neuronas espejo se active cuando el terapeuta se mueve en sincronía con el cliente o cuando simplemente lo observa, generando en ambos casos una conectividad interneuronal entre los dos individuos. Traduciéndolo al reflejo empático, éste permitiría la recogida de información del paciente durante la sesión además de facilitar la comunicación y el intercambio.

Una intervención a través de la DMT, puede llegar a ser un salto cualitativo en mujeres con cáncer de mama. No sólo puede permitir una mejor adaptación a la nueva situación de la paciente recién diagnosticada sino también puede ser una forma de sobrellevar la enfermedad tras un tratamiento farmacológico y quirúrgico.

A través de la DMT se pretende la toma de conciencia del cuerpo más allá de la fatiga y la enfermedad, fortaleciendo recursos físicos, psicológicos y emocionales. En definitiva, mejorar la calidad de vida, tanto en un diagnóstico reciente como tras años del mismo.

Aunque el hecho de ser un caso clínico puede verse como una limitación en cuanto a la validez de los datos, el bienestar percibido y expresado por la paciente a lo largo de las sesiones permite entrever la relevancia clínica de la $\mathrm{DMT}^{(50)}$. Teniendo en cuenta el feedback positivo sobre el bienestar subjetivo percibido por ella, una futura propuesta sería realizar dicha intervención en un contexto grupal, ampliando y diversificando el proceso empático al añadir al binomio terapeuta-paciente el de paciente-paciente. 


\section{Agradecimientos}

Queremos agradecer a la paciente participante su tiempo así como su valentía y gran generosidad al expresar sus vivencias.

\section{REFERENCIAS BILBIOGRÁFICAS}

1. Sandel SL, Judge JO, Landry N, Faria L, Ouellette, R, Majczak, M. Dance and movement program improves quality-oflife measures in breast cancer survivors. Cancer Nurs 2005;28:301-9.

2. Ho RTH. Effects of dance movement therapy on Chinese cancer patients: A pilot study in Hong Kong. Art Psychother 2005;32:33745. Doi: 10.1007/s10465-005-9002-z.

3. Molinaro J, Kleinfeld M, Lebed S. Physical therapy and dance in the surgical management of breast cancer. A clinical report. Phys Ther 1986;66:967-9.

4. Stanton Jones K. Movement and meaning. En: Dance Movement Therapy in Psychiatry. Londres: Routledge,1992; p. 59-80.

5. Wengrover $\mathrm{H}$, Chaiklin S. La vida es danza, Barcelona: Ed. Gedisa, 2008.

6. Schoop T, Mitchell P. Won't you join the dance? A dancer's essay into the treatment of psychosis. Oxford (England): National Press; 1974.

7. Rossberg-Gempton I, Poole G. (1992). The relationship between body movement and affect: From historical and current perspectives. Art Psychother 1992;19:3946. Doi: 10.1016/0197-4556(92)90062-S.

8. Stanton Jones K. Movement and meaning. In: . En: Payne H, editor. Dance Movement Therapy in Psychiatry. Londres, Routledge; 1992. p. 59-80.

9. Winnicott DW. Playing and reality. London: Tavistock Publications; 1971.

10. Todd M. The thinking body. Princeton (New Jersey): Princeton Book Company; 1937.

11. Dibbell-Hope $S$. The use of dance/movement therapy in psychological adaptation to breast cancer. Art Psychother 2000; 27:51-68. Doi: 10.1016/S0197-4556(99)00032-5.
12. Goodill S. An introduction to medical dance/movement therapy: Health care in motion. London: Jessica Kingsley Publishers, 2005.

13. Lippin RA, Micozzi MS. Arts Therapy. En: M. S. Micozzi, editor. Fundamentals of complementary and integrative medicine. St Louis, MO: Saunders, 2006; p. 332-50.

14. Aktas G, Ogce F. Dance as a therapy for cancer prevention. Asian Pac J Cancer Prev 2005;6:408-11.

15. Hanna JL. The power of dance: health and healing. J. Altern Complement Med 1995;1:323-31. Doi: 10.1089/ acm.1995.1.323.

16. Ginsburgs VH, Goodill SW. A dance/ movement therapy clinical model for women with gynecologic cancer undergoing high dose rate brachytherapy. Am J Dance Ther 2009; 31:136-58. Doi: 10.1007/s10465-009-9076-0

17. Vulcan M. Is there any body out there? A survey of literature on somatic countertransfrence and its significance for DMT. Art Psychother 2009;36:275-81. Doi: 10.1016/j.aip.2009.06.002.

18. Bloom K. The embodied self. London: Karnac, 2006.

19. Dosamantes-Beaudry I. Somatic transference and countertransference in psychoanalytic intersubjective dance/movement therapy. Am J Dance Ther 2007; 29:73-89. Doi: 10.1007/s10465-007-9035-6.

20. Lewis P. The creative arts in transference/ countertransference relationships. Art Psychother 1992;19:317-23. Doi: 10.1016/0197-4556(92)90027-L.

21. Meekums B. Embodiment in Dance/ Movement Therapy training and practice. En: Payne H, editor. Dance/Movement Therapy: Theory, research and practice. Hove: Brunner-Routledge, 2006. p. 167-84.

22. Meekums B. Sponaneous symbolism in clinical supervision: Moving beyond logic. Body, movement and dance in Psychotherapy 2007; 2:95-107. Doi: 10.1016/ j.aip.2009.06.002. 
23. Pallaro P. Somatic countertransference: The therapist in the relationship. En Pallaro $P$, editor. Authentic Movement. London: Jessica Kingsley Publishers, 2007; p. 176-93.

24. Shaw R. The embodied psychotherapist: an exploration of the therapists' somatic phenomena within the therapeutic encounter. Psychother Res 2004;14:27188. Doi: 10.1093/ptr/kph025.

25. Shaw R. Psychoterapist embodiment. Couns Psychoter J 2004;15:14-7.

26. Forester C. Body awareness: An aspect of countertranference management that moderates vicarious traumatization. Dissertation, California Institute of Integral Studies, 2000.

27. Berger-Roskin M. Bodily experience and expression of emotion, in toward a body of knowledge: A collection of early writings Columbia MD: American Dance Therapy Association, 1989; p. 152-91.

28. Dosamantes-Alperson E. Experiential movement psychotherapy. In Lewis P, editor. Theoretical approaches in dance/ movement. lowa: Kendall/Hunt, 1984; p. 145-65.

29. Rizzolatti G, Fabbri-Destro M, Cattaneo L. Mirror neurons and their clinical relevance. Nat Clin Pract Neurol 2009;5:24-34. Doi: 10.1038/ncpneuro0990.

30. Cattaneo L, Rizzolatti G. The mirror neuron system. Arch Neurol 2009,66:557-60. Doi: 10.1001/archneurol.2009.41.

31. Gallese V. Before and below theory of mind: Embodied simulation and the neural correlates of social cognition. Philos T Roy Soc B 2007;362:659-69. Doi: 10.1098/ rstb.2006.2002.

32. Decety J. L'empathie est-elle unie simulation de la subjectivité d'autrui? En: Berthoz A, Jorland G, editores. Paris: Odile Jacob, 2004. p. 53-89.

33. Buccino G, Luui F, Canessa N, Patteri I, Lagravinese G, Benuzzi F, et al. Neural circuits involved in the recognition of actions performed by nonconspecifics: an fMRI study. J Cognitive Neurosci 2004;16:11426. Doi: 10.1162/089892904322755601.

34. Iacoboni M, Molnar-Szakacs I, Gallese V, Buccino G, Mazziotta JC, Rizzolatti G. Grasping the intentions of others with one's owns mirror neuron system. PLOS Biology 2005;3:529-35. Doi: 10.1371/journal. pbio.0030079.

35. Newman-Norlund RD, van Schie HT, van Zuijlen AM, Bekkering $\mathrm{H}$. The mirror neurons system is more active during complementary compared with imitative action. Nat Neurosci 2007; 10:817-18. Doi: 10.1038/nn1911.

36. Iacoboni M, Woods RP, Brass M, Bekkering H, Mazziota JC, Rizzolatti B. Cortical mechanisms of human imitation. Science 1999;286:2526-8. Doi: 10.1126/ science. 286.5449.2526.

37. Melsom, A. M. Dance/movement therapy for psychosocial aspects of heart disease and cancer: An exploratory literature review. Unpublished Masters thesis, Hahnemann University, 1999.

38. Blazquez A, Guillamó E, Javierre C. Preliminary experience with Dance Movement Therapy in patients with chronic fatigue syndrome. Art Psychoter 2010; 37:285-92. Doi: 10.1016/j.aip.2010.05.003.

39. Petrone K. The role of dance/movement therapy in a cancer support group: A group case study. Unpublished Master's thesis, MCP Hahnemann University, 1997.

40. Konarski K. Theorell T. Psykosomatisk Läkekonst (Psychosomatic Medicine). Stockholm: Natur och Kultur, 1991.

41. Laban R, Danza educativa moderna. Barcelona: Paidós Ibérica, 1984.

42. PallaroP. Selfand body-self: dance/movement therapy and the development of object relations. Art Psychother 1996;23:113-9. Doi: 10.1016/0197-4556(95)00061-5.

43. Fawzy Fl. Psychosocial interventions for patients with cancer: what works and what doesn't. Eur J Cancer 1999;35:1559-64. Doi: 10.1016/S0959-8049(99)00191-4. 
44. Gallese V, Fadiga L, Fogassi L, Rizzolatti G. Action recognition in the premotor cortex. Brain 1996;119:593-609. Doi: 10.1093/ brain/119.2.593.

45. Gallese V. Intentional attunement: a neurophysilogical perspective on social cognition and its disruption in autism. Brain Res 2006; 1079: 15-24. Doi: 10.1016/j. brainres.2006.01.054.

46. Winters AF. Emotion, embodiment, and mirror neurons in dance/movement therapy: a connection across disciplines. Am J Dance Ther 2008;30:84-105. Doi: 10.1007/s10465-008-9054-y.

47. Carr L, lacoboni M, Dubeau MC, Mazziotta JC, Lenzi GL. Neural mechanisms of empathy in humans: A relay from neural systems for imitation to limbic areas. P Natl
Acad Sci USA 2003;100:5497-502. Doi: 10.1073/pnas.0935845100.

48. Stern DN. The interpersonal world of the infant: A view from psychoanalysis \& developmental psychology. New York: Basic Books, 1985/2000.

49. Berrol CF. Neuroscience meets dance/ movement therapy: mirror neurons, the therapeutic process and empathy. Art Psychother 2006; 33: 302-15. Doi: 10.1016/j.aip.2006.04.001.

50. Courneya KS, Friedenreich CM, Sela RA, Quinney HA, Rhodes RE, Handman M. The group psychotherapy and home-based physical exercise (group-hope) trial in cancer survivors: Physical fitness and quality of life outcomes. Psychooncology 2003; 12:357-74. Doi: 10.1002/pon.658. 
